\title{
RG scaling at chiral phase transition in two-flavor QCD
}

\author{
Ken-Ichi Ishikawa*† \\ Graduate School of Science, Hiroshima University, Higashi-Hiroshima, Hiroshima 739-8526, \\ Japan \\ E-mail: ishikawa@theo.phys.sci.hiroshima-u.ac.jp

\section{Yoichi Iwasaki} \\ Center for Computational Sciences, University of Tsukuba, Tsukuba, Ibaraki 305-8577, Japan \\ E-mail: iwasaki@ccs.tsukuba.ac.jp
}

\begin{abstract}
We investigate the nature of the chiral phase transition using the RG improved gauge action and the Wilson quark action with two degenerate quarks on $32^{3} \times 16,24^{3} \times 12$, and $16^{3} \times 8$ lattices. We introduce RG scaling relations for both the temporal and the spacial effective masses of mesons at the chiral phase transition point. Numerical results of effective masses at the chiral phase transition on the three sizes of lattices are excellently on the universal limiting curves for the pseudo-scalar meson and vector meson, respectively. The scaling enables us to obtain the effective masses in the continuum limit as functions of the distance. We also examine the case of massive quarks at the chiral transition. On each size of lattices a hyper-scaling relations is satisfied and find the anomalous mass dimension $\gamma^{*} \simeq 0.67-1.1$.
\end{abstract}

34th annual International Symposium on Lattice Field Theory

24-30 July 2016

University of Southampton, $U K$

\footnotetext{
* Speaker.

${ }^{\dagger}$ Poster
} 


\section{Introduction}

The properties of the chiral phase transition of QCD at high temperature are key ingredients for describing the early stage of the evolution of the Universe and for understanding the phenomena in the heavy ion collision experiments. It is still under debate the order of the chiral phase transition of two-flavor of QCD, first- or second-order phase transition.

We have proposed new RG scaling relations for the conformal theories with an infrared fixed point [1]. We extend the RG scaling relation to the problem of the chiral phase transition of twoflavor of QCD.

We employ the RG-improved gauge action and two-flavor of Wilson fermion action. Two point correlation functions of the pseudo-scalar and vector mesons are investigated on the lattices of $32^{3} \times 16,24^{3} \times 12$, and $16^{3} \times 8$ at the chiral phase transition point. The critical point on each lattice is identified as

$$
\begin{aligned}
& \beta \simeq 2.8, \quad K_{c}=0.1455 \quad \text { on } 32^{3} \times 16, \\
& \beta \simeq 2.6, \quad K_{c}=0.149 \quad \text { on } 24^{3} \times 12 \text {, } \\
& \beta \simeq 2.3, \quad K_{c}=0.1547 \quad \text { on } 16^{3} \times 8 \text {, }
\end{aligned}
$$

by monitoring the number of iteration of CG inversion. The phase transition temperature is estimated to be $T_{c} \simeq 163\left({ }_{-1}^{+11}\right) \mathrm{MeV}$ from the hadron spectroscopy on a $16^{3} \times 64$ lattice.

\section{RG scaling relations}

In the vicinity of a critical point, RG relations for the two point correlation functions are given as follows;

$$
\begin{aligned}
& G_{t}\left(n_{t} ; g, m_{q}, N_{s}, N_{t}, \mu\right)=\left(\frac{N^{\prime}}{N}\right)^{-2 \gamma} G_{t}\left(n_{t}^{\prime} ; g^{\prime}, m_{q}^{\prime}, N_{s}^{\prime}, N_{t}^{\prime}, \mu^{\prime}\right), \\
& G_{s}\left(n_{s} ; g, m_{q}, N_{s}, N_{t}, \mu\right)=\left(\frac{N^{\prime}}{N}\right)^{-2 \gamma} G_{s}\left(n_{s}^{\prime} ; g^{\prime}, m_{q}^{\prime}, N_{s}^{\prime}, N_{t}^{\prime}, \mu^{\prime}\right) .
\end{aligned}
$$

Here $\mu^{\prime}=\mu / s$ and $N_{s}^{\prime}=N_{s} / s, N_{t}^{\prime}=N_{t} / s$, and $n^{\prime}=n / s$. The UV renormalization scale $\mu$ in lattice theories is set by the inverse lattice spacing $a^{-1}$. Note $N_{s} a=L_{s}$ and $N_{t} a=L_{t}$ are kept constant. The relation between $g^{\prime}$ and $g$ and $m_{q}^{\prime}$ and $m_{q}$ are determined by the beta function $\mathscr{B}$ and the mass anomalous dimension $\gamma$.

In the case of an IR fixed point $g^{*}$, we may suppress $g=g^{*}$, as well as $m_{q}=0$, and $\mu$ ( $\mu$ is determined from $N ; 1 / a=N / L)$.

$$
\tilde{G}_{t}(\tau, N)=G_{t}\left(n_{t}, N\right), \quad \text { with } \quad \tau \equiv n_{t} / N .
$$

The variable $n_{t}$ takes $0,1,2, \cdots, N_{t}-1$ so that $0 \leq \tau \leq 1$. Thus the scaling relation at the IR fixed point becomes

$$
\tilde{G}_{t}(\tau, N)=\left(\frac{N^{\prime}}{N}\right)^{-2 \gamma^{*}} \tilde{G}_{t}\left(\tau, N^{\prime}\right),
$$


with $N, N^{\prime} \rightarrow \infty$.

We further simplify the scaling relations in terms of the scaled effective mass $\mathfrak{m}_{t}(\tau)$ defined by

$$
\mathfrak{m}_{t}\left(n_{t}\right)=N \ln \frac{G_{t}\left(n_{t}, N\right)}{G_{t}\left(n_{t}+1, N\right)}
$$

which is reduced to

$$
\mathfrak{m}_{t}(\tau)=-\partial_{\tau} \ln \tilde{G}_{t}(\tau, N)
$$

From Eqs. (2.4) and (2.6) we obtain

$$
\mathfrak{m}_{t}(\tau, N)=\mathfrak{m}_{t}\left(\tau, N^{\prime}\right) .
$$

We applied the new scaling relation Eq. (2.7) in order to identify the IR fixed point for $N_{f}=$ $16,12,8,7$ in $S U$ (3) gauge theories in Ref. [1]. In this paper we will extend the RG scaling relation to the chiral phase transition.

Now let us consider RG transformation at finite temperature $T$. Note the RG transformation keeps automatically the temperature $T=1 / L_{t}=1 / N_{t} a$ constant. In the vicinity of the chiral phase transition point the RG equation for the temporal propagator is given by

$$
\mathfrak{m}_{t}(\tau, g, N)=\mathfrak{m}_{t}\left(\tau, g^{\prime}, N^{\prime}\right),
$$

and similarly for the spatial propagator.

We will also consider the RG relations in the massive case, with the coupling constant $g$ at the chiral transition point. The RG relations for the spatial effective masses is given by

$$
\mathfrak{m}_{x}(\tau, g, m, N)=\mathfrak{m}_{x}\left(\tau, g^{\prime}, m^{\prime} N^{\prime}\right) .
$$

In the limit $N, N^{\prime} \rightarrow \infty, m_{q}^{\prime}=s^{y} m_{q}$ with $y=1+\gamma^{*}$.

\section{Results}

Figs 1 and 2 show the scaled effective masses $\mathfrak{m}(\tau)$ for the pseudo-scalar and vector mesons at the critical point. All simulation points of Eq. (1.1) are overlaid in the same figure. We observe the data points from the spatial correlation functions (Fig. 1) are excellently on the scaling curve except three points at short distance $\left(n_{x}=0,1,2\right)$. The temporal effective masses (Fig. 2) are consistent with the scaling at the long distance range. Note all four panels of figures show results consistent with scaling without adjustable parameters.

The scaling Eq. (2.7) implies

$$
m\left(t ; g, m_{q}=0, N, \mu\right)=\frac{\mathfrak{F}_{\mathfrak{s}}(\tau)}{N} .
$$

The $\mathfrak{F}_{\mathfrak{s}}(\tau)$ is a function independent from $g$ and $N$. In the limit $N \rightarrow \infty, m$ vanishes as $1 / N$. It may be emphasized that we may obtain the continuum limit as a function of $\tau$. 

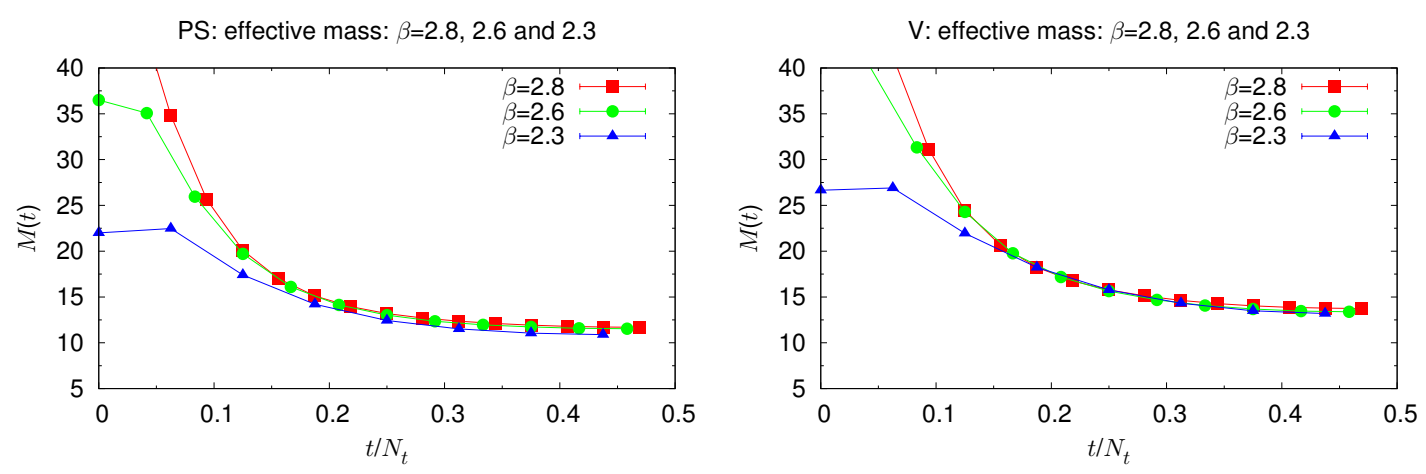

Figure 1: Effective mass plot for the spatial two-point functions (left:ps mesons, right: vector mesons).
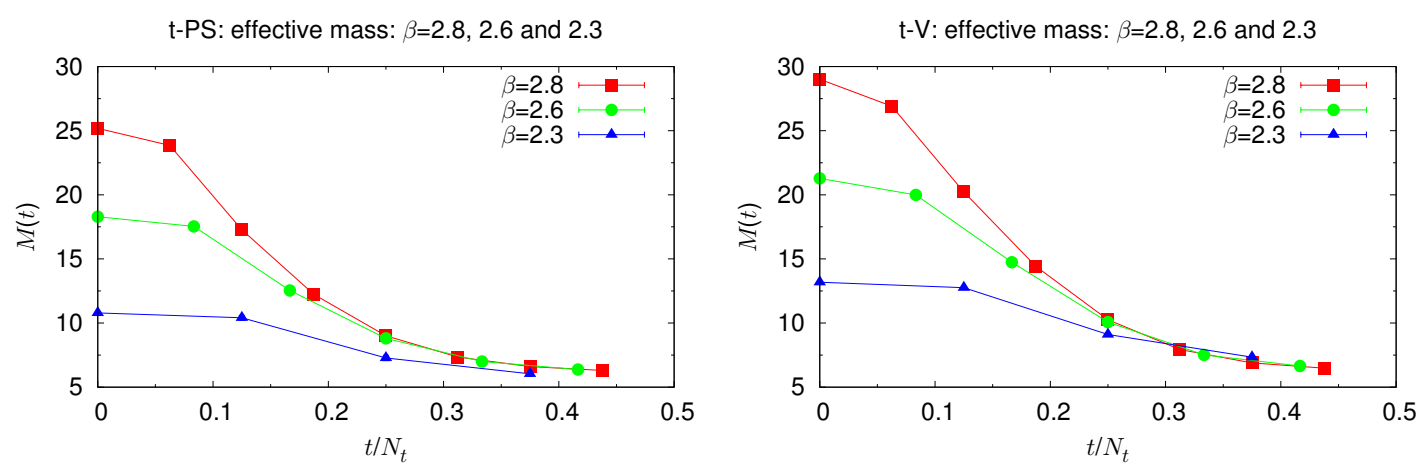

Figure 2: Effective mass plot for the temporal two-point functions (left:ps mesons, right: vector mesons).

We see the effective masses given in Fig. 1 monotonously decreasing functions. This is the point emphasized in Ref. [2]. The effective mass shows a power modified Yukawa type exponential form. The fit with $\hat{m}=0.478$ and $\alpha=0.7854$ agrees well with data $10 \leq n_{x} \leq 15$.

Now let us discuss the massive cases. We plot the $\log \left(m_{\mathrm{PS}}\right)$ vs. $\log \left(m_{q}\right)$ in Fig. 3. The data are excellently on the linear lines at each of lattice sizes $N=16,24,32$. We fit $m_{\mathrm{PS}}$ as a function of $m_{q}$ with

$$
\log \left(m_{\mathrm{PS}}\right)=d \log \left(m_{q}\right)+c .
$$

This implies a hyper scaling relation with anomalous mass dimension $\gamma^{*}$ at each lattice size

$$
m_{\pi} \propto m_{q}^{d}
$$

with $d=1 /\left(1+\gamma^{*}\right)$ [3]. Table 1 shows the anomalous mass dimension at each $N$. The $N$ dependence of the anomalous mass dimension is linear in terms $1 / N$. We fit $\gamma^{*}$ linearly in $1 / N$ and obtain

$$
\gamma^{*}=0.19(4), \quad \chi^{2} / \mathrm{DoF}=0.0056
$$




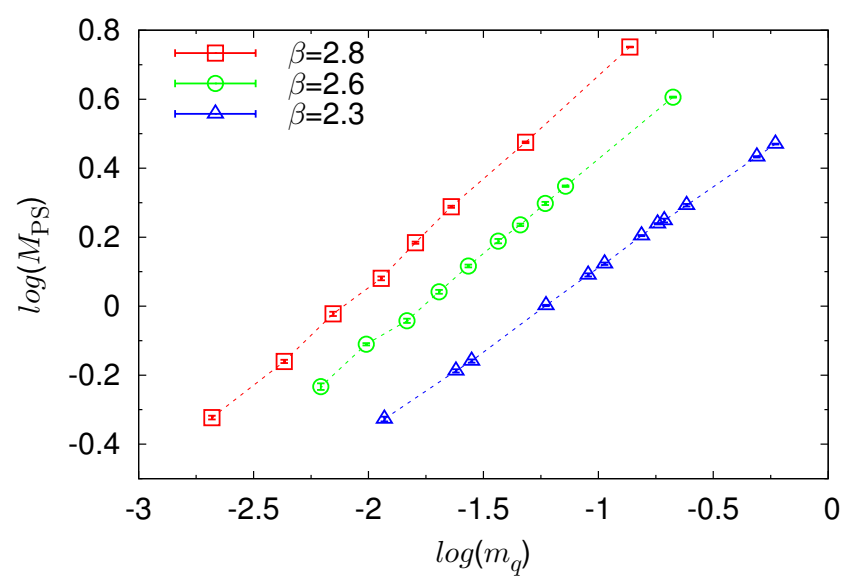

Figure 3: $\log$ (quark mass) vs. $\log$ (meson mass)

\begin{tabular}{ccc}
\hline \hline$N$ & $d$ & $\gamma^{*}$ \\
\hline 16 & $0.468(1)$ & $1.14(4)$ \\
24 & $0.545(2)$ & $0.83(7)$ \\
32 & $0.600(2)$ & $0.667(6)$ \\
\hline \hline
\end{tabular}

Table 1: Anomalous mass dimension extracted from the hyper-scaling relation.

\section{Summary}

In this poster we presented a first attempt to apply the RG scaling relations we proposed [2] to investigation of the chiral phase transition of two-flavor QCD using lattice method. After identifying the chiral phase transitions on three sizes of lattices, we introduce several RG scaling relations. The scaling enables us to obtain the effective masses in the continuum limit as functions of the distance. We also examine the case of massive quarks at the chiral transition. On each size of lattices a hyper-scaling relations is satisfied and find the anomalous mass dimension $\gamma^{*} \simeq 0.67-1.1$.

We would like to investigate the $T$ dependence of physical quantities in the near future. Both of $m_{q}$ and $T$ dependences of effective masses will provide us several critical exponents.

\section{Acknowledgments}

We would like to thank Yu Nakayama and T. Yoshie for useful discussion. The calculations were performed with HA-PACS computer at CCS, University of Tsukuba and SR16000 at KEK. We would like to thank members of CCS and KEK for their strong support for this work.

\section{References}

[1] K.-I. Ishikawa, Y. Iwasaki, Y. Nakayama and T. Yoshie, Phys. Lett. B 748, 289 (2015) doi:10.1016/j.physletb.2015.07.019 [arXiv:1503.02359 [hep-lat]].

[2] K.-I. Ishikawa, Y. Iwasaki, Y. Nakayama and T. Yoshie, Phys. Rev. D 87 (2013) no.7, 071503 doi:10.1103/PhysRevD.87.071503 [arXiv:1301.4785 [hep-lat]]. 
[3] L. Del Debbio and R. Zwicky, Phys. Rev. D 82 (2010) 014502 doi:10.1103/PhysRevD.82.014502 [arXiv:1005.2371 [hep-ph]]. 